weeks 1, 4, 12, 24 and 48 of follow-up (FU). At diagnosis, all patients received $\mathrm{GC}$ in line with EULAR recommendations with a slow GC tapering starting after week 4. At week 12, some of the patients (16/27) received additionally leflunomide $(10 \mathrm{mg})$. Whole blood samples were stained, lysed, fixed and analyzed by flow cytometry. The expression of adhesion molecules (CD62L, CD11b) was determined on neutrophils. Sera levels of serum amyloid A (SAA) and IL-6 were measured by nephelometry and ELISA, respectively. Levels of IL-8, IL-18, IL-23, L-selectin and $\mathrm{CHI} 3 \mathrm{~L} 1$ were determined by MagPix using human pre-mixed multi-analyte kits.

Results: At weeks 1 and 4 of FU we detected a decrease in neutrophil expression of CD62L and CD11b, as well as in sera levels of SAA, IL6, IL-8, IL-18, L-selectin and CHI3L1 in all GCA patients. At week 12 (8 weeks after GC tapering) an elevation of CD11b, SAA, IL-6 and IL-23 as compared to week 4 was observed (Figures 1 and 2). At weeks 24 and 48 of FU we identified four different groups of biomarkers. The first group consisted of neutrophil CD62L $(\mathrm{p}<0.05)$ and serum IL-6, that showed a marked increase in patients receiving GC therapy only, while decreasing in patients receiving GC in combination with leflunomide. The second group is represented by neutrophil CD11b and serum IL-8 that were higher at week 24 in the GC-treated group (the first FU after receiving leflunomide), however their levels were equal at week 48 in both groups of GCA patients (Figures 1 and 2). In the third group, the serum levels of SAA, IL-18 and L-selectin decreased at week 24 and remained stable through week 48 , regardless of therapy used. The fourth group of biomarkers included serum IL-23 and CHI3L1, levels of which declined at weeks 24 and 48 in patients receiving GC only and substantially increased in patients receiving leflunomide and GC (Figure 2).

Conclusion: Neutrophil surface markers CD62L and CD11b together with inflammatory parameters (SAA, IL-6, IL-8 and IL-23) could represent informative biomarkers for monitoring disease progression in GCA patients. Important biomarker differences were observed between GC-treated GCA patients, in the presence and absence of leflunomide, serving as a good basis for predicting relapses.
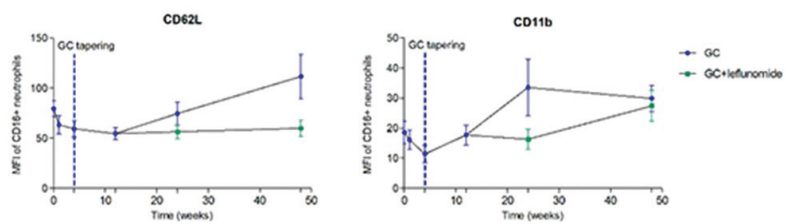

Abstract THU306 - Figure 1. Longitudinal L-selectin (CD62L) and integrin $\alpha M$ (CD11b) surface expression on neutrophils from GCA patients at T0 (prior to therapy) and 1, 4, 12, 24 and 48 weeks after therapy. Shown is mean \pm SEM. At week 12, 16/27 GCA patients received leflunomide, in addition to GC therapy.
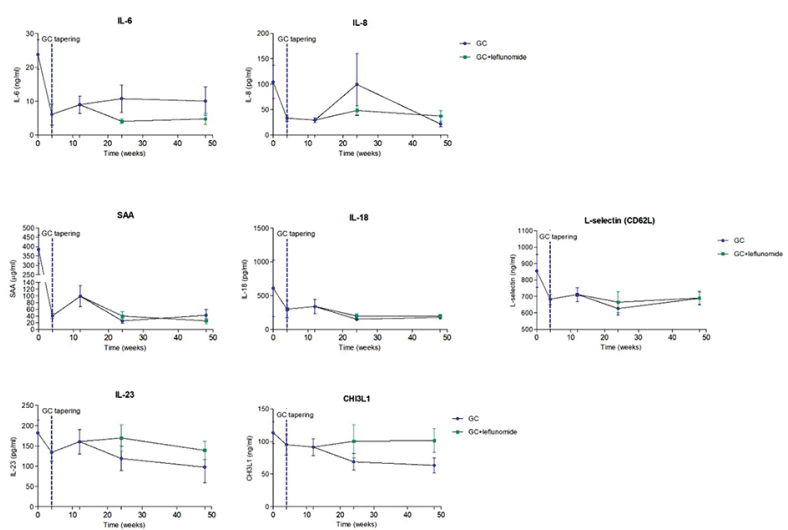

Abstract THU306 - Figure 2. Serum levels of IL-6, IL-8, SAA, IL-18, L-selectin, IL-23 and CHI3L1 in longitudinally followed GCA patients at T0 (prior to therapy) and 4, 12, 24 and 48 weeks after therapy. Shown is mean \pm SEM. At week 12, 16/27 GCA patients received leflunomide, in addition to GC therapy.

Acknowledgement: The authors would like to acknowledge funding from the Slovenian Research Agency (ARRS) for the National Research Program P30314.

Disclosure of Interests: None declared

DOI: 10.1136/annrheumdis-2019-eular.4136

\section{THU0307 \\ THE HALO SIGN IN THE EMERGENCY DEPARTMENT: COMPLEMENTARY VALUE TO THE APPLICATION OF THE CRITERIA FOR THE CLASSIFICATION OF GIANT CELL ARTERITIS IN AN ACUTE ENVIRONMENT}

Carlos Guillén-Astete, Fernando Lopez-Gutierrez, Ana Rodriguez-García, Carmen Larena, Monica Luque-Alarcón, Jose Luis Morell Hita. Hospital Ramón Y Cajal, MADRID, Spain

Background: In a previous study (Acta Reum 2018; Vol.4.No.1:5), we identified the low diagnostic value of the criteria for classification of giant cell arteritis (GCA) in patients with such suspicion in an emergency setting of a third level hospital. In some emergency units, linear probe ultrasound equipment is available for immediate use. It would be of interest to determine whether the training of healthcare personnel in halo sign recognition could be useful in addition to the CGA criteria in situations of suspicion.

Objectives: We present a sub-study in which we incorporate the ultrasound study of temporal atheria (TA) as an added criterion to determine its contribution in terms of diagnostic validation.

Methods: We reviewed the casuistry of patients who consulted the emergency department with different combinations of the rest of the ACG classification criteria, included in the aforementioned study. The entry requirements were: Age > 40 years and headache as a reason for consultation. We identified those patients who underwent temporal artery ultrasound during assessment before receiving corticoid therapy. The pattern of comparison was the definitive diagnosis of ACG (biopsy).

Results: Thirty cases of ACG were identified by compatible biopsy that had an ultrasound study of AT in the emergency department. On the other hand, 47 ultrasound AT studies were identified in patients who were not finally diagnosed with ACG. All included records were distributed between 2012 and 2016. Ultrasound exploration of positive AT was understood as that with 2 or more branches with positive halo sign (Sifuentes, Ann Rheum Dis 2013-eular- OP0208). Tables 2x2 were elaborated to establish the results of the validation test with three and four ACG classification criteria. One patient was identified with the combination of criteria: cephalea $+V S G>50 \mathrm{~mm} / \mathrm{h}+$ Alt. of the exploration of the AT and 4 with cephalea + Age $>50$ years + Alt. of the exploration of the AT. Of these patients, only one had ultrasound study of AT so they were excluded from the study. With 4 criteria + halo sign $(+)$, an $S$ of $93.3 \%$ and NPV of $93.3 \%$ was achieved; with 3 criteria + halo sign $(+$ an $\mathrm{S}$ of $83.3 \%$ and NPV of $93.7 \%$ was achieved. The attached table shows all the results of the validation test.

\section{Abstract THU0307 -Table 1}

CRITERIA COMBINATION

\begin{tabular}{l|llll}
\hline HEADACHE + AGE > 50 YEARS + ESR > 50 + & $94.1 \%$ & $35.4 \%$ & $56.2 \%$ & $93.6 \%$ \\
$\begin{array}{l}\text { TEMPORAL ARTERY ANORMALIT) } \\
\text { 4 CRITERIA + HALO (+) }\end{array}$ & $82.3 \%$ & $93.3 \%$ & $93.3 \%$ & $82.3 \%$ \\
$\begin{array}{l}\text { HEADACHE + AGE > 50 YEARS + ESR > } \\
\text { 5OMM/H }\end{array}$ & $96.9 \%$ & $25.7 \%$ & $38.3 \%$ & $94.5 \%$ \\
3 CRITERIA + HALO (+) & $76.9 \%$ & $93.7 \%$ & $83.3 \%$ & $90.9 \%$
\end{tabular}

Conclusion: Although the application of the GCA classification criteria has insufficient specificity and low positive predictive value, when adding the ultrasound study of TA, specificity exceeds 93\% and PPV exceeds $93 \%$ when combined with 4 criteria and $83 \%$ when combined with 3 criteria Although our results should be supported by a larger sample size, our findings assume that the routine use of this technique would contribute to improving the diagnostic accuracy of this disease and our ability to initiate treatment in a more timely manner.

Disclosure of Interests: None declared

DOI: 10.1136/annrheumdis-2019-eular.8170

\section{THU0308 ASSESSMENT OF SEXUAL AND PSYCHOLOGICAL STATES OF BEHCSET'S DISEASE MALE PATIENTS}

Alaa A A Mohamed ${ }^{1}$, Manal Hassanien ${ }^{1}$, Amr Abou Faddan ${ }^{2}$, Arwa Aljohi ${ }^{1}$, Safaa A Mahran $1 .{ }^{1}$ Assiut University Hospital, Rheumatology and Rehabilitation, Assiut, Egypt, ${ }^{2}$ Assiut University Hospital, Urology, Assiut, Egypt

Background: Behçet's disease (BD) is a chronic multisystem autoimmune disease characterized by orogenital ulceration, uveitis, skin lesions and vascular involvement of different organs in the body. The sexual and psychological aspects of the disease are still not fully recognized.

Objectives: We aimed to evaluate the sexual and psychological status of $\mathrm{BD}$ male patients and to assess the relationship between the sexual function and depression in this cohort. 
Methods: The patients, fulfilling the International Criteria for Behçet's disease (ICBD), and healthy controls, of a comparable age, were evaluated in the Rheumatology and Urology Clinics. The demographic and clinical data was collected. Disease activity of the patients was assessed using Behçet's Syndrome Activity Score (BSAS). For both groups, the sexual function and the psychological status were evaluated using the International Index of Erectile Functions (IIEF- 5) and the Beck Depression Inventory (BDI), respectively. Spearman's rho, Chi-Square and Mann-Whitney $U$ tests were used as appropriated.

Results: The number of BD patients was 26 and that of the controls was 27. The mean age of the patients was $36 \pm 7.4$ and that of controls was $40.9 \pm 5.2$. Though BD patients were younger in age they exhibited significantly lower scores in sexual function, and scored higher in depression as illustrated in Table 1.

Abstract THU308 - Table 1. Demographic and clinical data of the participants

\begin{tabular}{lccc}
\hline & $\begin{array}{c}\text { Patients } \\
(\mathrm{n}=26)\end{array}$ & $\begin{array}{c}\text { Controls } \\
(\mathrm{n}=27)\end{array}$ & P value \\
\hline Smoking, $\mathrm{n}(\%)$ & $8(30.8 \%)$ & $4(14.8 \%)$ & 0.4 \\
Disease duration (years), mean $\pm \mathrm{SD}$ & $4.2 \pm 1.9$ & $\mathrm{NA}$ & $\mathrm{NA}$ \\
$\begin{array}{l}\text { Erectile dysfunction (IIEF-5 score), median } \\
\text { (Interquartile range) }\end{array}$ & $15(9-20)$ & $20(20-25)$ & $<0.001^{*}$ \\
$\begin{array}{l}\text { Depression score (Beck questionnaire), median } \\
\text { (n) }\end{array}$ & $13(9-18)$ & $9(7-14)$ & $0.039^{*}$
\end{tabular}

(Interquartile range)

There were no associations between the disease duration, smoking status or different clinical phenotypes and the sexual or psychological functions. However, there was an inverse relationship between BSAS and the sexual function (rho $=-.443, p=.024)$. The duration of corticosteroids intake was negatively correlating with the sexual function ( $r h o=-.604, p=.001$ ) as well. The depression scores correlated significantly with the presence of inflammatory back pain ( $\mathrm{rho}=.413, \mathrm{p}=.036$ ).

There was no association between the severity of sexual dysfunction and that of depression. The patients showed different degrees of depression, and there was a tendency towards increased rates of erectile dysfunction in all groups as shown in Table 2.

Abstract THU308 - Table 2. Correlation of IIEF-5 and BDI

\begin{tabular}{|c|c|c|c|c|c|}
\hline \multirow{2}{*}{$\begin{array}{l}\text { Sexual function } \\
\text { (IIEF-5 score) }\end{array}$} & \multicolumn{3}{|c|}{ Beck Depression Inventory (BDI) } & \multirow[t]{2}{*}{ Total } & \multirow{2}{*}{$\begin{array}{c}\mathrm{P} \\
\text { value }\end{array}$} \\
\hline & $\begin{array}{l}\text { Minimal } \\
(\mathrm{n}=14)\end{array}$ & $\begin{array}{l}\text { Mild } \\
(\mathrm{n}=7)\end{array}$ & $\begin{array}{c}\text { Moderate } \\
(\mathrm{n}=5)\end{array}$ & & \\
\hline $\begin{array}{l}\text { No ED or Mild ED } \geq 17, n \\
(\%)\end{array}$ & $4(28.6 \%)$ & $\begin{array}{c}3 \\
(42.9 \%)\end{array}$ & $2(40 \%)$ & 9 & 0.78 \\
\hline$E D \leq 16, n(\%)$ & $10(71.4 \%)$ & $\begin{array}{c}4 \\
(57.1 \%)\end{array}$ & $3(60 \%)$ & 17 & \\
\hline
\end{tabular}

ED; erectile dysfunction.

Conclusion: BD has a negative impact on men's psychological state and sexual function. Severe disease states, evident in the long duration of corticosteroids use and the high activity scores, are linked to sexual dysfunction.

Disclosure of Interests: None declared

DOI: 10.1136/annrheumdis-2019-eular.4326

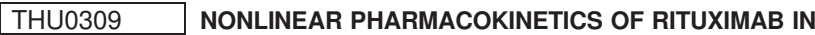 ANTI-NEUTROPHIL CYTOPLASMIC ANTIBODY ASSOCIATED VASCULITIS}

Denis Mulleman ${ }^{1,2}$, Amina Bensalem ${ }^{1}$, David Ternant ${ }^{1,3}$, Gilles Paintaud ${ }^{1,3}$, Divi Cornec $^{4,5}$, Ulrich Specks ${ }^{6}$, The rituximab vs cyclophosphamide for ANCAassociated vasculitis (RAVE) trial. ${ }^{1}$ University of Tours, EA 7501 GICC, Tours, France; ${ }^{2} \mathrm{CHRU}$ de Tours, Service de Rhumatologie, Tours, France; ${ }^{3} \mathrm{CHRU}$ de Tours, Laboratoire de Pharmacologie-Toxicologie, Tours, France; ${ }^{4}$ University of Brest, Inserm UMR1227 LBAI, Brest, France, ${ }^{5} \mathrm{CHRU}$ de Brest, Service de Rhumatologie, Brest, France; ${ }^{6}$ Mayo Clinic, Division of Pulmonary and Critical Care Medicine, Rochester, United States of America

Background: Rituximab (RTX) was approved in patients with anti-neutrophil cytoplasmic antibody (ANCA) associated vasculitis (AAV).

Objectives: To describe the pharmacokinetics of rituximab in AAV patients and to explore its sources of variability
Methods: Rituximab pharmacokinetics was described in 92 AAV patients from the RAVE trial (rituximab for ANCA- associated vasculitis) using a population modeling approach. A semi-mechanistic model including a latent target antigen turnover allowed the estimation of specific targetmediated elimination of rituximab in addition to its non-specific elimination Sex, body surface area (BSA), BVAS/WG score, and status newly diagnosed on pharmacokinetic parameters were investigated as covariates. Results: A two compartments model including target mediated elimination best described rituximab pharmacokinetics. The mean (interindividual standard deviation) estimated central volume of distribution was $3.06 \mathrm{~L}$ $(2.61 \%)$, systemic clearance was $0.135 \mathrm{~L}_{\text {days }}{ }^{-1}(6.43 \%)$, and targetmediated elimination rate constant was $19.13 \times 10^{-6} \mathrm{nmol}^{-1}$ days ${ }^{-1}$ (fixed to 0 ). The initial activity of the disease was higher in male than in female $\left(p=4.09 \times 10^{-14}\right)$, and in patients newly diagnosed at inclusion $\left(p=7.91 \times 10^{-5}\right)$. Moreover, systemic clearance was slightly lower in granulomatosis with polyangiitis group than in other AAV groups $\left(p=3.6 \times 10^{-}\right.$ $\left.{ }^{3}\right)$. Neither BVAS/WG score nor BSA were significant covariates in a multivariate analysis.

Conclusion: We report a nonlinear target-mediated elimination of rituximab in AAV patients. Granulomatosis with polyangiitis patients have a lower global clearance of rituximab than other AAV groups. Our study not sup port the utility of BSA based individualized dosing protocols, as previously reported (1)

\section{REFERENCE:}

[1] Cornec D, et al. Pharmacokinetics of rituximab and clinical outcomes in patients with anti-neutrophil cytoplasmic antibody associated vasculitis. Rheumatology. 2018;57:639-50.

Disclosure of Interests: Denis Mulleman Speakers bureau: Pfizer, Novartis, Grifols, Amina Bensalem: None declared, David Ternant Speakers bureau: Sanofi, Amgen, Gilles Paintaud Grant/research support from: Novartis, Roche Pharma, Sanofi-Genzyme, Chugai, Pfizer and Shire, Divi Cornec: None declared, Ulrich Specks: None declared DOI: 10.1136/annrheumdis-2019-eular.4897

\section{THU0310 OPTIMAL INITIAL DOSE OF GLUCOCORTICOID FOR ELDERLY-ONSET ANCA ASSOCIATED VASCULITIS: SAFTY OUTOCOME ANALYSIS OF TWO NATIONWIDE, PROSPECTIVE, INCEPTION COHORT STUDIES}

Keiji Ohashi ${ }^{1}$, Kenei Sada ${ }^{1}$, Yosuke Asano ${ }^{1}$, Keigo Hayashi $^{1}$, Sumie Hiramatsu Asano' ${ }^{1}$, Yuriko Yamamura ${ }^{1}$, Michiko Morishita', Haruki Watanabe ${ }^{1}$, Mariko Narazaki ${ }^{1}$, Yoshinori Matsumoto ${ }^{1}$, Tomoko Kawabata ${ }^{1}$, Jun Wada ${ }^{1}$, Masayoshi Harigai ${ }^{2}$, Hirofumi Makino ${ }^{3}$, On behalf of Japan Research Committee of the Ministry of Health, Labour, and Welfare for Intractable Vasculitis Syndrome (JPVAS). 'Okayama University Graduate School of Medicine, Dentistry and Pharmaceutical Sciences, Department of Nephrology, Rheumatology, Endocrinology and Metabolism, Okayama, Japan; ${ }^{2}$ School of Medicine, Tokyo Women's Medical University, Department of Rheumatology, Tokyo, Japan; ${ }^{3}$ Okayama University, Okayama, Japan

Background: Glucocorticoid (GC) is still the mainstay of treatment for antineutrophil cytoplasmic antibody (ANCA) -associated vasculitis (AAV) while GC use, disease severity, and older age are risk factors for accrual of damage and infection in $A A V^{1 / 2)}$.

Objectives: To explore optimal initial dose of GC for patients with elderly-onset AAV based on safety outcome analysis using data from two nationwide prospective inception cohort studies (RemIT-JAV and RemITJAV-RPGN).

Methods: RemIT-JAV and RemIT-JAV-RPGN enrolled consecutive patients with newly diagnosed AAV fulfilling the criteria for primary systemic vasculitis as proposed by the European Medicines Agency algorithm and requiring immunosuppressive treatment. From the cohort studies, elderlyonset ( $\geq 65$ years) patients with microscopic polyangiitis and granulomatosis with polyangiitis, classified as generalized or severe disease type according to the European Vasculitis Study Group-defined disease severity, were enrolled in this analysis. The primary outcome measures were Vasculitis Damage Index (VDI) at $24^{\text {th }}$ month and serious infections during 2 years after starting treatment for AAV. The patients were divided into three groups based on initial dose of GC: high-dose (HD) group, prednisolone $(\mathrm{PSL}) \geq 0.8 \mathrm{mg} / \mathrm{kg} /$ day; medium-dose group (MD), $0.6 \leq \mathrm{PSL}$ $<0.8 \mathrm{mg} / \mathrm{kg} /$ day; low-dose (LD) group, PSL $<0.6 \mathrm{mg} / \mathrm{kg} /$ day. The VDI were classified into treatment-related $\mathrm{VDI}^{1}{ }^{1}$ and disease-related VDI 